\title{
Enterprise in Physical Science
}

\begin{abstract}
Summary
With an increasing emphasis on enterprise and innovation in government directives, it is timely to consider the embedding of enterprise activities within the physical science curriculum. We have piloted a course "Physics in an Enterprise Culture", and are now expanding to embrace project work and dual degree schemes.
\end{abstract}

Subject area: Physical Science

\section{Description}

The work is concerned with embedding and enhancing enterprise skills in the undergraduate curriculum through option courses, project work and ultimately dual degree schemes.

\section{Type of activity}

Currently an option module is available at level 3: "Physics in an Enterprise Culture". Dual degrees (BSc and MPhys) will come on stream in September 2003 "Physics (or Chemistry) with Enterprise Management".

Group project work at level 3.

Summer placements in Sheffield or Silicon Valley.

\section{Content covered}

Project Proposal: research proposal mechanisms in UK academia. Why are formal proposals used? The EPSRC "standard" proposal form and Case for Support .Making your proposal for a Laboratory Experiment. Peer review. Prioritisation.

Patents, Protection and Marketing: What is a patent? Why are patents needed? Is a good idea enough? How is a prototype developed and marketed? What makes a commercially successful product?

Product Dissection: Have you ever wondered what is inside a walkman? Or a mobile phone? Or a camera? What were the challenges in putting these components together? This theme involves the "dissection" of a product to find out how it operates and what its limitations are. What opportunities remain for further development? Case studies.

\section{Application}

This model is transferable to any Physical Science discipline, with appropriate choice of exemplars.

Many physical science graduates go in to the SME environment, which requires the rapid application of new knowledge in highly competitive fields. This brief exposure provides students with the essentials of what they need to know for such a working environment.

\section{Further comments}

The option course has proved very popular with our students. They found the process of project proposal and refereeing very revealing in terms of how to persuade others 
New Directions in the Teaching of Physical Sciences

of the merit of your own case, and how to critically review the suggestions of others. The product dissection gave students the opportunity to think how the core physics studied has been used in modern day technology, to realise the limitations that remain to be overcome (e.g. battery technology), and the highly competitive market place. We found it of immense value to bring in a physics entrepreneur to meet with the students and present a case study of his own company.

It has also been important to have under continual development a web site of resources which may be used to seek out information and stimulate ideas.

\section{Author(s)}

Professor M R J Gibbs

Department of Physics \& Astronomy

University of Sheffield

\section{Contact details}

Professor Mike Gibbs or Dr Tim Richardson

Department of Physics \& Astronomy

University of Sheffield

Sheffield

S3 7RH

M.R.Gibbs@Shef.ac.uk_T.Richardson@Shef.ac.uk

\section{URL}

http://www.shef.ac.uk/uni/academic/N-Q/phys/teaching/phy335/ 